\title{
Performance of primary care physicians on early skin cancer diagnosis (melanoma and non- melanoma)
}

\begin{abstract}
Skin cancer is a matter of public health concern, since melanoma is an aggressive and potentially lethal skin tumor, and non-melanoma skin cancer is the most common type of cancer in the world population. This way, it's a role of generalist and primary care physicians perform early diagnosis and refer to dermatologists. In this present comment, we aim to discuss this role, demonstrating the accuracy and showing new perspectives for early diagnosis and correct referrals.
\end{abstract}

Keywords: skin cancer, primary care physicians, early diagnosis
Volume 3 Issue 4 - 2019

Dimitri Luz Felipe da Silva, Lorena

Visentainer,Andréa Eloy Fernandes da Costa

França, Renata Ferreira Magalhães

Dermatology department, University of Campinas, Brazil

Correspondence: Dimitri Luz Felipe da Silva, Rua Vital Brasil, cidade universitária, Hospital de clínicas, secretaria de dermatologia, Brazil, Tel +551935217776,

Email dimitriluzfs@gmail.com

Received: April 30, 2019 | Published: August 30, 2019

\section{Introduction}

Non-melanoma skin cancer (basal cell and spinal cell carcinoma) are responsible for more than $98 \%$ of skin cancer cases, being the most common type of cancer in the American population and worldwide. Melanoma corresponds to the 19th most common type of cancer in both genders (excluding non-melanoma skin cancer) and present a high mortality rate. ${ }^{1,2}$

Despite recognizing dermatology as the specialty more liable for diagnosis and treatment of skin cancer, pointed by $95 \%$ of primary care physicians (PCP), ${ }^{3}$ the access and referrals to these specialists can take long and can be expensive for the system, making necessary for PCP's to be able to do the right diagnosis, which would lead to an increase in the rate of early diagnosis in skin cancer and would lower the rate of unnecessary referrals, leading to economy.

There is a lack of articles regarding sensitivity and specificity rates in diagnosing a melanoma at full clinical checks performed by a general physician, but the United States preventive services task force (USPSTF) has published some data showing a sensitivity range $42-100 \%$ in the year of 2009 , then about 2 articles were published on this scope, and the new USPSTF recommendation in 2016 showed a decrease at the range (40-70\%). The specificity range went from 70-98\% (USPSTF 2009) to 86-98\% (USPSTF 2016). ${ }^{2,4}$ Further methodologic reviews did not provide similar data for epithelial tumors.

It is known that dermatologists have higher rates, as shown in studies with a sensitivity rate around $74.1-89.4 \%$, increasing accordingly with groups that analyses dermatoscopic images in addition to the clinics, when dealing with melanoma. ${ }^{5}$ PCPs can perform regular skin checks, but the lack of training to recognize potential malignancies is a challenge that is not easily solved only with educational programs and algorhitms. ${ }^{6}$

Among patients that had a clinical skin examination by a general doctor, it was showed that $18 \%$ of the patients referred to a dermatologist needed a biopsy to exclude the diagnosis of a skin cancer (non-melanoma and pre-cancers), $34,2 \%$ of patients seen by a PCP received the right diagnosis of skin cancer or actinic keratosis. ${ }^{7}$ This study also showed a high rate of referrals that could be avoided if other ways of making diagnosis were used, even though it also shows that educational intervention with the doctors was not effective in increasing early detection diagnosis, also there was a lack of data and similar studies.

This shows us the importance of a complementary tool to support PCP to increase their sensibility and avoid unnecessary biopsies and surgeries, and to help the dermatologists on decisions. Teledermatology could be used, giving hand of this tool, to increase early diagnosis in unassisted populations.

\section{Acknowledgment}

None.

\section{Funding}

None.

\section{Conflicts of interest}

The authors report no conflicts of interest.

\section{References}

1. Bray F, Ferlay J, Soerjomataram I, et al. Global Cancer Statistics 2018. 2019.

2. Bibbins-Domingo K, Grossman DC, Curry SJ, et al. Screening for skin cancer: US Preventive Services Task Force recommendation statement. JAMA. 2016;316(4):429-435.

3. Ibrahimi OA, Bangash H, Green L, et al. Perceptions of expertise in cutaneous surgery and cosmetic procedures: what primary care physicians think. Dermatol Surg. 2012;38(10):1645-1651;

4. Wolff T, Tai E, Miller T. Screening for skin cancer: an update of the evidence for the US Preventive Services Task Force. Ann Intern Med. 2009;150(3):194-198. 
5. TJ Brinker, Heckler A, Hauschild A, et al. Comparing artificial intelligence algorithms to 157 German dermatologists: the melanoma classification benchmark. Eur Journal of Cancer. 2019;111:30-37;

6. Shaub AR, Lewis ET, Swetter SM, et al. Physician Perceptions of Primary Care-based Skin Cancer Screening in a Veterans Affairs Pilot Study. Br J Dermatol. 2019;181(2):377-378.
7. Swetter SM, Chang J, Shaub AR et al. Pilot feasibility study of primary care-based skin cancer screening in a Veterans Affairs Health Care System. JAMA Dermatol. 2017;153(8):797-801. 